\title{
Comparative studies on osmosis based encapsulation of sodium diclofenac in porcine and outdated human erythrocyte ghosts
}

\author{
Katarina Bukara ${ }^{a, b, *}$, Ivana Drvenica ${ }^{b}, V^{2}$ esna Ilićc ${ }^{c}$, Ana Stančićc ${ }^{c}$, Danijela Mišićc ${ }^{d}$, \\ Borislav Vasićc $^{e}$, Radoš Gajićc ${ }^{e}$, Dušan Vučetić ${ }^{f}$, Filip Kiekens ${ }^{a}$, Branko Bugarski ${ }^{b}$ \\ a Department Pharmaceutics, Campus Drie Eiken, University of Antwerp, Antwerp, Belgium \\ ${ }^{\mathrm{b}}$ Department of Chemical Engineering, Faculty of Technology and Metallurgy, University of Belgrade, Belgrade, Serbia \\ ${ }^{\mathrm{c}}$ Institute for Medical Research, University of Belgrade, Belgrade, Serbia \\ d Institute for Biological Research “Siniša Stanković”, University of Belgrade, Bulevar Despota Stefana 142, 11060 Belgrade, Serbia \\ e Institute of Physics, University of Belgrade, Pregrevica 118, 11080 Zemun, Belgrade, Serbia \\ ${ }^{\mathrm{f}}$ Institute for Tranfusiology and Haemobiology of Military Medical Academy, Belgrade, Serbia
}

\section{A R T I C L E I N F O}

\section{Article history:}

Received 10 March 2016

Received in revised form 16 October 2016

Accepted 19 October 2016

Available online 20 October 2016

\section{Keywords:}

Pharmaceutical biotechnology

Slaughterhouse blood

Erythrocyte membranes

Encapsulation

Gradual hypotonic hemolysis

Controlled drug delivery

\begin{abstract}
A B S T R A C T
The objective of our study was to develop controlled drug delivery system based on erythrocyte ghosts for amphiphilic compound sodium diclofenac considering the differences between erythrocytes derived from two readily available materials - porcine slaughterhouse and outdated transfusion human blood. Starting erythrocytes, empty erythrocyte ghosts and diclofenac loaded ghosts were compared in terms of the encapsulation efficiency, drug releasing profiles, size distribution, surface charge, conductivity, surface roughness and morphology. The encapsulation of sodium diclofenac was performed by an osmosis based process - gradual hemolysis. During this process sodium diclofenac exerted mild and delayed antihemolytic effect and increased potassium efflux in porcine but not in outdated human erythrocytes. FTIR spectra revealed lack of any membrane lipid disorder and chemical reaction with sodium diclofenac in encapsulated ghosts. Outdated human erythrocyte ghosts with detected nanoscale damages and reduced ability to shrink had encapsulation efficiency of only $8 \%$. On the other hand, porcine erythrocyte ghosts had encapsulation efficiency of $37 \%$ and relatively slow drug release rate. More preserved structure and functional properties of porcine erythrocytes related to their superior encapsulation and release performances, define them as more appropriate for the usage in sodium diclofenac encapsulation process.
\end{abstract}

(c) 2016 Elsevier B.V. All rights reserved.

\section{Introduction}

Sodium diclofenac (SD) is a potent non-steroidal antiinflammatory drug with analgesic and antipyretic effects. However, due to its physicochemical action on the gastric mucous (Oddsson et al., 1990) and inflammatory action on the small bowel and the colon (Carson et al., 1990; Witham, 1991), it counts for a risk factor of relatively high incidence of gastrointestinal side effects. Because of the mentioned side effects and its short biological halflife (Todd and Sorkin, 1988), SD is an ideal candidate for prolonged release preparations with the aim to maintain therapeutic activity, reduce toxic effects and improve patient compliance (Krajišnik

\footnotetext{
* Corresponding author at: Department Pharmaceutics, Campus Drie Eiken, University of Antwerp, Antwerp, Belgium.

E-mail addresses: kbukara@tmf.bg.ac.rs, katarinabukara@gmail.com (K. Bukara).
}

et al., 2013). Many chemically- synthetized or naturally occurring carrier materials such as phospholipid liposomes (Lopes et al., 2004), sodium alginate beads (Kulkarni et al., 1999), PLGA-based nanoparticles (Cooper D and Harirforoosh, 2014), silica-chitosan composites (Kozakevych et al., 2013), solid lipid nanoparticles (Liu et al., 2014) have been employed to design micro- or nano- sized prolonged/controlled delivery systems for SD. However, in general, the surface chemistry of the mentioned carriers differs substantially from the chemistry of the cell membrane (Mao et al., 2011). Studies conducted on liposomes showed that SD due to its surfaceactive properties can incorporate into membranes affecting the lipidic organization and leading to solubilisation of the structure (Lopes et al., 2004). On the other hand, erythrocyte membrane, which is more complex than liposomal membrane and also interact with SD via class of lipids found in its outer moiety (Suwalsky et al., 2009), but without the disruption, might be a naturally occurring alternative offering potential advantage in comparison with the 
liposomes as drug carriers. However, to the best authors' knowledge, literature data regarding erythrocyte based delivery systems for SD is very scarce and there hasn't been any attempt since Jain et al. developed magnetically responsive SD loaded erythrocytes (Jain and Vyas, 1994).

The majority of research groups are dealing with active substances encapsulation in small amount of autologous erythrocytes intended for personal medicine. Having in mind the ultimate goal of universal erythrocyte membrane based SD carrier production, the use of non-infectious blood material generally treated as waste, such are slaughterhouse blood and outdate transfusion human blood represent beneficial approach in the development of an added-value product. This approach is supported by the increasing number of emerging techniques for covering antigenic determinants (Mansouri et al., 2011), which could allow application of these materials as carriers and the fact that these materials are available in unlimited amounts. Thus, we have recently shown that osmosis based process-gradual hemolysis can be used for loading dexamethasone sodium phosphate on large scale into erythrocytes membranes (i. e. ghosts) derived from porcine and bovine slaughterhouse blood (Kostić et al., 2014).

In xenotransplantation research, a pig accounts for the favoured available source of organs and tissues (Doucet et al., 2004). Therefore, in this study we examined the gradual hypotonic hemolysis as an osmosis based SD encapsulation process in erythrocyte membranes derived from two inexpensive, available in sufficient amount materials-porcine slaughterhouse and outdated human blood. Obtained formulations with encapsulated SD were characterized in terms of encapsulation efficiency, drug release profile, morphology, size and surface properties.

\section{Materials and methods}

\subsection{Erythrocyte samples}

Porcine slaughterhouse blood and human outdated blood were used as a starting biological material for erythrocytes isolation. Porcine blood was from the slaughterhouse "PKB Imes" in Belgrade, Serbia. Transport and treatment of the Swedish Landrace swine in the slaughterhouse was in obedience to the National Regulation on Animal Welfare, and performed in compliance with institutional animal care and use policies. The porcine blood collection and transport was done according to the protocol described in Kostic et al. (Kostić et al., 2014). The human erythrocytes concentrates were from Institute for Transfusiology and Hemobiology, Military Medical Academy, Belgrade, Serbia. The blood was from healthy blood donors. The erythrocytes were enriched by the standard procedure and preserved in SAGM solution (each $100 \mathrm{~mL}$ contains $0.900 \mathrm{~g}$ dextrose monohydrate, $0.877 \mathrm{~g}$ sodium chloride, $0.0169 \mathrm{~g}$ adenine, and $0.525 \mathrm{~g}$ mannitol) for 42 days at $4{ }^{\circ} \mathrm{C}$. The outdated cell packs were anonymized prior to distribution, and the link to the donor was broken. If were not used for the research, these cell packs would be discarded after the expiration date. Preparation of packed porcine and human erythrocytes suspensions were performed as described in Kostić et al. (Kostić et al., 2014).

\subsection{Erythrocyte ghosts' preparation and encapsulation (loading) with $S D$}

In order to prepare erythrocyte ghosts, porcine and human erythrocytes were undertaken to the osmosis based process-gradual hypotonic hemolysis (Kostić et al., 2014).

According to known literature data regarding the different osmotic properties of porcine and human erythrocytes (Kostić et al., 2014; Doucet et al., 2004), hypotonic $35 \mathrm{mM}$ sodium phos- phate/ $\mathrm{NaCl}$ buffer for porcine and $5 \mathrm{mM}$ sodium phosphate buffer for human erythrocytes were introduced gradually for $30 \mathrm{~min}$ with flow rate $150 \mathrm{~mL} / \mathrm{h}$ in $50 \mathrm{~mL}$ of packed erythrocytes in PBS (with hematocrit value of 0.5 ) and continuously mixed. After the hemolysis the erythrocyte ghosts were isolated, washed three times in hypotonic solution used in gradual hemolysis process and finally in isotonic PBS solution.

For the purposes of encapsulation, gradual hypotonic hemolysis process was performed as previously described but in the presence of SD in concentration of $120 \mathrm{mg} / \mathrm{L}$. After the hemolysis, the erythrocyte ghosts were precipitated ( 40 min centrifugation at $3220 \times \mathrm{g}$, at $4{ }^{\circ} \mathrm{C}$ ), supernatant containing hemoglobin was discarded and erythrocyte ghosts were washed four times in the buffer solution used for the gradual hypotonic hemolysis process. Densely packed erythrocyte ghosts in the volume of $2 \mathrm{~mL}$ were incubated with $8 \mathrm{~mL}$ of PBS containing $120 \mathrm{mg} / \mathrm{L} \mathrm{SD}, 90 \mathrm{~min}$ at $37^{\circ} \mathrm{C}$. After the incubation, samples were stored at $4{ }^{\circ} \mathrm{C}$ over the night. The next day, erythrocyte ghosts were precipitated by $40 \mathrm{~min}$ centrifugation at $3220 \times \mathrm{g}$. The erythrocyte ghosts in the volume of $220 \mu \mathrm{L}$ were dispersed in test tubes containing $1780 \mu \mathrm{L}$ of hypertonic 2 XPBS solution with SD $(120 \mathrm{mg} / \mathrm{L})$ and $0.01 \%$ glutaraldehyde and then incubated for $30 \mathrm{~min}$ at $37^{\circ} \mathrm{C}$ in test tubes. The encapsulated ghosts were precipitated by 5 min centrifugation at $10,000 \times \mathrm{g}$ and washed out twice in PBS solution without SD, to remove non-encapsulated SD.

\subsection{Sample preparation for qualitative and quantitative analysis of encapsulated (loaded) SD}

From the final suspension of encapsulated erythrocyte ghosts, $100 \mu \mathrm{L}$ was taken and dispersed in $2 \mathrm{~mL}$ of distilled water and mixed with $2 \mathrm{~mL}$ of methanol. Test tubes were stored at the room temperature for $30 \mathrm{~min}$ and then centrifuged $15 \mathrm{~min}$ at $14,000 \times \mathrm{g}$. Obtained supernatants were filtered through the filters with pore size $0.2 \mu \mathrm{m}$ into the vials and used for the below described HPLC analysis.

\subsection{Qualitative and quantitative analysis of encapsulated $S D$}

SD is quantified in samples of loaded ghosts using an UHPLC/DAD/-HESI-MS/MS method. Separation, identification, and quantification of diclofenac was performed using Dionex Ultimate 3000 UHPLC system (ThermoFisher Scientific, Bremen, Germany) coupled to diode array detector (DAD), and connected to TSQ Quantum Access Max triple-quadrupole mass spectrometer (ThermoFisher Scientific, Basel, Switzerland). Elution was performed at $30^{\circ} \mathrm{C}$ on Hypersil gold $\mathrm{C} 18$ column $(50 \times 2.1 \mathrm{~mm})$ with $1.9 \mu \mathrm{m}$ particle size (ThermoFisher Scientific, USA). The mobile phase consisted of $(A)$ water $+0.1 \%$ formic acid, and (B) acetonitrile (MS grade, Fisher Scientific UK, Leics, UK), which were applied in the gradient elution previously described in Kostić et al. (Kostić et al., 2014) The flow rate was set to $0.4 \mathrm{~mL} \mathrm{~min}^{-1}$ and the detection wavelengths to 230 and $280 \mathrm{~nm}$. The injection volume was $10 \mu \mathrm{L}$. All the analyses were performed in triplicate.

Mass spectrometer was equipped with an heated electrospray ionization (HESI) source, and was used with vaporizer temperature kept at $350^{\circ} \mathrm{C}$, and ion source settings as follows: spray voltage $3500 \mathrm{~V}$, sheet gas (N2) pressure $28 \mathrm{AU}$, ion sweep gas pressure $0 \mathrm{AU}$ and auxiliary gas (N2) pressure at $4 \mathrm{AU}$, capillary temperature at $270^{\circ} \mathrm{C}$, skimmer offset $0 \mathrm{~V}$. Mass spectrometry data were acquired in negative mode, in $\mathrm{m} / \mathrm{z}$ range from 100 to 1000 . Collision-induced fragmentation experiments, including product ion scanning (PIS) and selected reaction monitoring (SRM), were performed using argon as the collision gas, and collision energy (cE) was set to $20 \mathrm{eV}$. SRM experiment for quantitative analysis was performed by using two MS2 fragments, which were previously defined as dominant in PIS experiments. Quantification of SD was based on the calibration curve of pure compound. Total amount of SD in each sample 
was evaluated by the calculation of peak areas, and is expressed as $\mathrm{ng} \mathrm{ml}^{-1}$. Chromeleon Xpress softver (Thermo Fisher Scientific, Bremen, Germany) was used for used for the data acquisition and method/run control.

\subsection{Loading parameters}

Two parameters were determined and were used for evaluation of loading efficiency of the method (Hamidi et al., 2011):

1. Loaded amount: The amount of SD encapsulated in $0.1 \mathrm{~mL}$ of the final packed erythrocyte ghosts.

2. Encapsulation efficiency: The percent ratio of loaded amount of SD to amount added per $0.1 \mathrm{~mL}$ during the encapsulation process.

\subsection{SD effect on gradual hypotonic hemolysis}

In order to investigate the effect of SD on gradual hypotonic hemolysis process, the $1 \mathrm{~mL}$ aliquots of the erythrocyte suspension were sampled at the beginning of the process, and in $1 \mathrm{~min}$ time interval for the first $5 \mathrm{~min}$ of hemolysis, in $3 \mathrm{~min}$ time intervals for the next $15 \mathrm{~min}$, and in the time intervals of $5 \mathrm{~min}$ by the end of the experiment.

As a control, the same test was performed in gradual hypotonic hemolysis processes without SD. The samples were centrifuged at $15000 \mathrm{~g}$ for $20 \mathrm{~min}$, and hemoglobin and potassium content in the supernatants were determined by cyanmethemoglobin method (Van Assendelft et al., 1984) and ion selective electrode, respectively. The percent of hemoglobin and potassium release were determined in reference to the total amount released at the end of gradual hypotonic hemolysis (100\% hemolysis).

\subsection{Particle size analysis}

Particle size and size distribution analysis of starting erythrocytes and resulting empty and SD loaded ghosts was performed by using laser-based particle size analyser Mastersizer Hydro $2000 \mathrm{~S}$ (Malvern Instruments Ltd, U.K.). For this purpose, the samples were analysed while suspended in isotonic PBS in a suitable concentration, according to instrument operation conditions.

\subsection{FT-IR analysis}

The FT-IR spectra of SD, lyophilized empty and SD loaded erythrocyte membranes were obtained by means of Fourier transform infrared spectroscopy (FTIR spectrometer Bomem MB, series Hartmann \& Braun) with a resolution of $2 \mathrm{~cm}^{-1}$, by the $\mathrm{KBr}$ method. The spectra were taken in the wavelength region $4000-400 \mathrm{~cm}^{-1}$ at room temperature.

\subsection{Zeta potential determination}

Zeta potential determination of starting erythrocytes, empty and encapsulated human and porcine erythrocyte ghosts were performed using a Zetasizer Nano ZS (Malvern Instruments, U.K.) apparatus operating at $\lambda=633 \mathrm{~nm}$ produced by an He-Ne laser at scattering angle $173^{\circ}$ at $25 \pm 0.1^{\circ} \mathrm{C}$. The calculation of zeta potential values of diluted samples (according to the manufacturer instructions) were done by computer software from electrophoretic mobility using the Smoluchowski equation: $\xi=\mu \eta / \varepsilon$, where $\xi$ is the zeta potential, $\mu$ is the mobility, $\eta$ is the viscosity of the solution, and $\varepsilon$ is the dielectric constant of the solvent. The results represent the average value of three consecutive measurements for each sample.

\subsection{Atomic force microscopy}

In order to perform morphological examination by AFM, the samples were fixed onto the surface of a coverslip by incubation with $2.5 \%$ glutaraldehyde in PBS for $1 \mathrm{~h}$. The samples were further fixed with osmium tetroxide $(0.1 \%)$ in PBS for $1 \mathrm{~h}$. Osmium tetroxide was removed by washing twice in PBS. The drying of samples was performed with an increasing concentrations of ethanol (10\%, 30\%, 50\%, 70\%, 95\% and 100\%) and finally in acetone. Topographic AFM measurements were performed in the semi-contact mode under ambient conditions using the NTEGRA Prima system from NT-MDT (NT-MDT Co. Moscow, Russia). NSG01 probes from NT-MDT with a typical tip curvature radius of about $6 \mathrm{~nm}$ and a typical force constant of $5 \mathrm{~N} / \mathrm{m}$ were used. Topographic images were processed using the instrument-equipped software by the plane subtraction or by the fitting with the first order lines. For the calculation of the root-mean-square (RMS) of the surface roughness, only cell surfaces were selected from AFM images and then RMS was directly generated by the instrument software, using a total of 10 cell measurements for each ghost preparations.

\subsection{Drug release}

To examine the release behaviour of SD from porcine and outdated human erythrocyte ghosts, $100 \mu \mathrm{L}$ of densely packed suspension in each test tube was diluted to $1 \mathrm{~mL}$ by adding $900 \mu \mathrm{L}$ of PBS solution. The tubes $(n=5)$ were mixed on the orbital shaker at $240 \mathrm{rpm}$ and incubated at $37^{\circ} \mathrm{C}$. After $1,2,24,48$ and $96 \mathrm{~h}$ one of the samples was harvested and after centrifuging at $10000 \mathrm{~g}$ for $10 \mathrm{~min}, 100 \mu \mathrm{L}$ of the supernatant was used for the HPLC analysis. These experiments were carried out in triplicate and the percent of SD release was determined in reference to a completely lysed sample ( $100 \%$ release). Due to possible microbial contamination drug releasing test was not performed longer than 4 days.

\subsection{Statistical analysis}

Experimental results were mean \pm SD of at least three measurements. Statistical analysis of the data regarding antihemolytic effect of SD, number based diameter distribution and $\zeta$-potential was performed by Student $t$-test with the Origin 9.0 Statistical Analysis Software. The values $\mathrm{p}<0.05$ were regarded as significant.

\section{Results and discussion}

In this study, encapsulation of SD into porcine and outdated human erythrocyte ghosts was performed by osmosis based process, gradual hypotonic hemolysis. As previously shown (Kostić et al., 2014; Stojanović et al., 2012) during the process of gradual decrease of osmotic strength of a solution surrounding erythrocytes, erythrocytes swell and hemoglobin molecules leak out. The transiently appeared membrane holes of $20-50 \mathrm{~nm}$ in the remaining erythrocyte membranes permit different extracellular substances to be encapsulated through these resealable pores (Fun et al., 2012). Beside already known phenomenon that different osmotic properties of the examined erythrocytes require the usage of different hypotonic conditions during encapsulation process, in this study the effect of the active substance-SD itself was examined on the encapsulation process, as well as on final properties of the encapsulated ghosts.

In order to identify and quantify encapsulated SD in a complex systems such as erythrocyte ghost, we developed simple, fast and reliable UHPLC/DAD/-HESI-MS/MS method. The analysis included comparison of the retention times, UV and MS/MS spectra with the available standard. Initially, full-scan UHPLC/-HESI-MS spectrum of pure SD was acquired in negative ion mode (Fig. 
1SB-Supplementary material 1) simultaneously with UHPLC/DAD spectrum at $\lambda=230$ and $280 \mathrm{~nm}$ (Fig. 1SD-Supplementary material 1). Peak at $\mathrm{Rt}=8.30 \mathrm{~min}$ corresponding to $\mathrm{SD}$, showed characteristic deprotonated molecule $m / z[\mathrm{M}-\mathrm{H}]^{-}$at 294, and $\lambda_{\max }$ at 230 and $280 \mathrm{~nm}$. The UHPLC/-HESI-MS/MS analysis, with cE set to $20 \mathrm{eV}$, revealed the major $-\mathrm{MS}^{2}$ product ions of $\mathrm{SD}: m / z\left[\mathrm{M}-\mathrm{H}-\mathrm{CO}_{2}\right]^{-}$ at $250, m / z\left[\mathrm{M}-\mathrm{H}-\mathrm{CO}_{2}-\mathrm{Cl}\right]^{-}$at 214 and $\mathrm{m} / z\left[\mathrm{M}-\mathrm{H}-\mathrm{CO}_{2}-2 \mathrm{Cl}\right]^{-}$at 178. UHPLC/-HESI-MS/MS PIS chromatogram and corresponding $-\mathrm{MS}^{2}$ spectrum is shown in Fig. 2SA1 and 2SA2-Supplementary material 2. Proposed fragmentation pattern in negative ion mode is also presented. Two characteristic -MS $\mathrm{Mr}^{2}$ fragments, $\mathrm{m} / \mathrm{z}$ at 250 and $\mathrm{m} / \mathrm{z}$ at 214, were used in SRM experiment for the accurate identification and quantification of SD (Fig. 2SA3 and 2SA4-Supplementary material 2) in human and porcine ghosts (Fig. 2SB). Developed UHPLC/DAD/-HESI-MS/MS method allowing simple, precise and accurate determination and quantification of the SD encapsulated in erythrocyte ghost demonstrated its ability to be used as analytical method in potential biomedical/clinical use of these SD carriers.

The amount of the encapsulated SD in $100 \mu \mathrm{L}$ of erythrocyte ghosts was $21.5 \mu \mathrm{g}$ and $4.85 \mu \mathrm{g}$ for porcine and outdated human samples, respectively. It corresponds to the encapsulation efficiency of $37 \%$ for porcine and $8.5 \%$ for outdated human erythrocyte ghosts. After the second washing out step during the encapsulation procedure (performed to remove all un-encapsulated drug), the detected amount of SD in the supernatant was $1.15 \%$ and $75.5 \%$ of the encapsulated amount for porcine and outdated human erythrocyte ghosts, respectively. The encapsulation efficiency for porcine erythrocyte ghosts under the applied conditions was consistent with the range reported in other studies where erythrocyte ghosts were used to encapsulate various agents such as drugs, enzymes, peptides, proteins, pollysacharides and DNA (Hamidi and Tajerzadeh, 2001; Millan et al., 2004). Co-encapsulation methods or enriched medium which could be a source of nutrients for erythrocyte (such as Hank's balanced salt solution) were not used in this study, so encapsulation efficiency was only the result of intrinsic ability of erythrocytes' membranes to entrap the substance. On the other hand, the encapsulation efficiency for outdated human erythrocyte ghosts was much lower than the reported range for fresh human erythrocytes (Hamidi and Tajerzadeh, 2001; Millan et al., 2004). This finding might be explained by the aging process and consequent deterioration of erythrocytes during the storage. During aging/storage erythrocytes lose water, ATP, hemoglobin, proteins, surface $\mathrm{N}$-acetylneuraminic acid and undergo age-related changes in band 3 (Huang et al., 2011). All these changes together and especially changes in band 3 which participate in hypotonic hemolysis process (Orhan and Sahin, 2001) were probably responsible for the decreased ability of outdated human erythrocytes to entrap the drug.

It was noted that with the applied encapsulation process, SD loaded porcine erythrocyte ghosts retained more hemoglobin in comparison with the empty erythrocyte ghosts. UV/VIS spectrophotometric measurements confirmed this observation revealing that the concentration of the residual hemoglobin in some of SD loaded porcine erythrocyte ghosts was even 3-4 folds higher than in the suspension of corresponding empty erythrocyte ghosts. On the other hand, the level of residual hemoglobin in outdated human SD loaded and empty erythrocyte ghosts was almost equal $(0.50 \%$ and $0.46 \%$ respectively). This was an incentive to investigate the effect of SD on the gradual hypotonic hemolysis process.

As it is shown in Fig. 1, during the gradual hypotonic hemolysis SD had only weak antihemolytic effect on porcine erythrocytes. $\mathrm{H} 50$ values (the point when $50 \%$ of total hemoglobin is released from erythrocytes) were $5.3 \pm 0.73$ and $5.84 \pm 0.5 \mathrm{~min}$ for erythrocytes suspension lysed in the hypotonic buffer without and with $S D$, respectively, and they didn't show statistically significant difference $(p>0.05)$. However during the washing out procedure as a
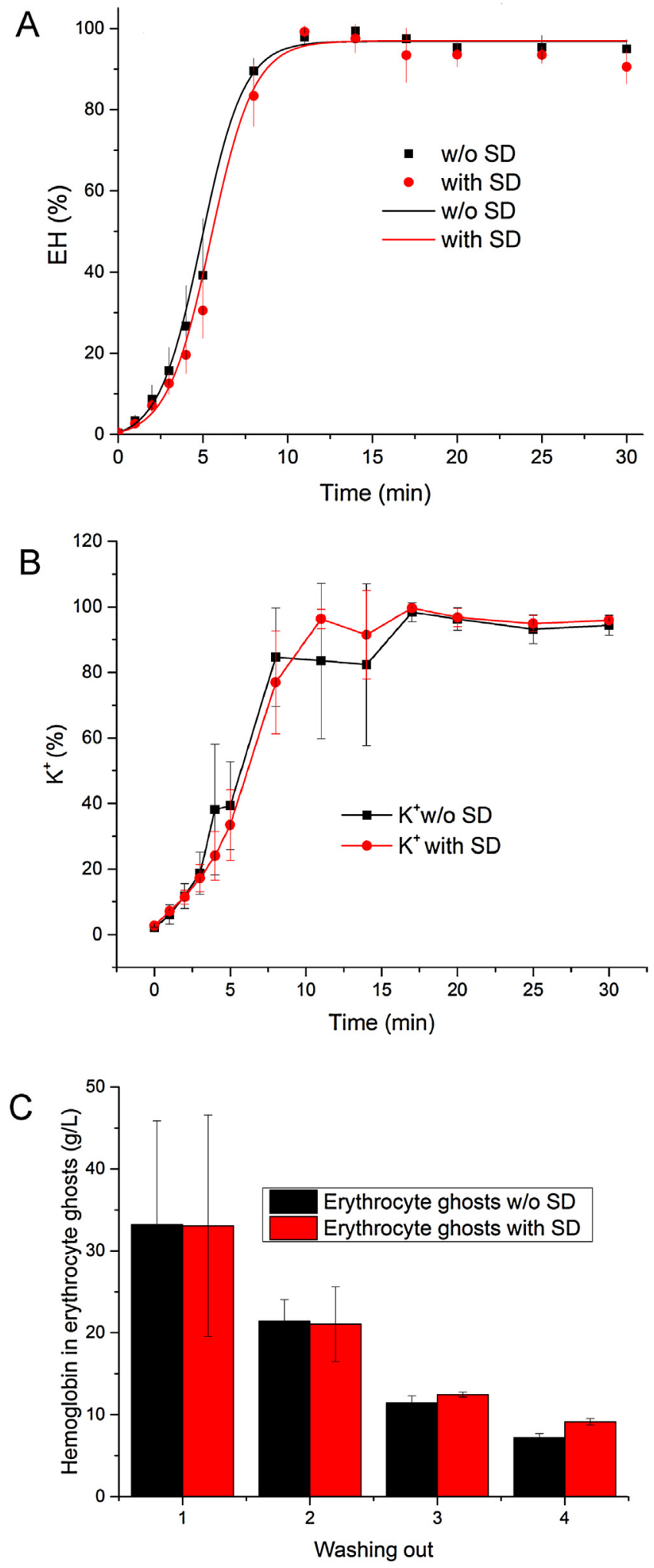

Fig. 1. Effect of SD on: (A) the gradual hypotonic hemolysis, (B) potassium efflux during the gradual hypotonic hemolysis $(C)$ washing out procedure of porcine erythrocyte ghosts. Results are mean \pm SD of three experiments. 

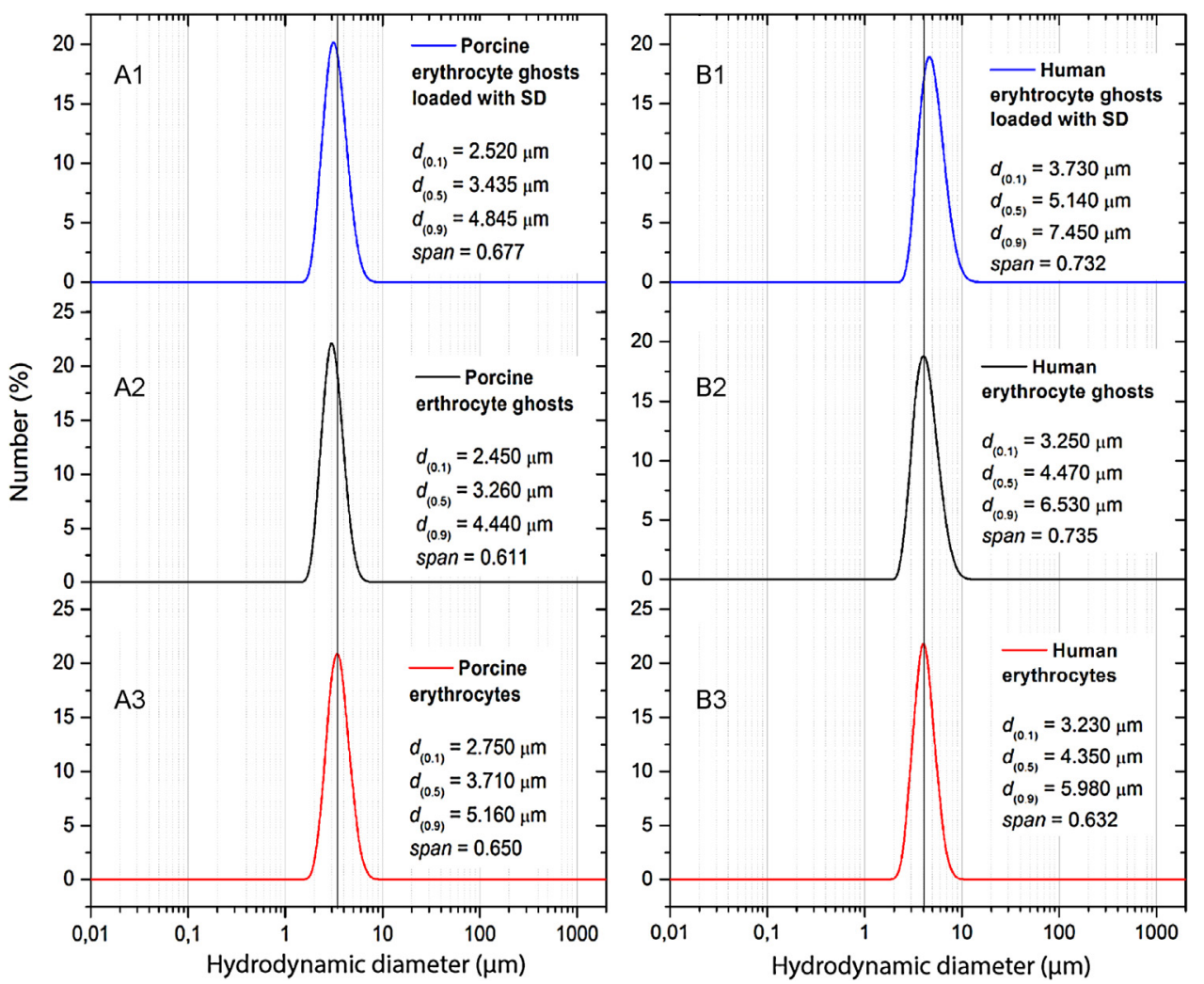

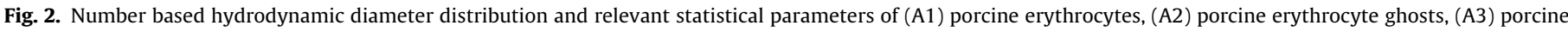
erythrocyte ghosts loaded with SD, (B1) outdated human erythrocytes, (B2) outdated human erythrocyte ghosts (B3) outdated human erythrocyte ghosts loaded with SD. ( $p>0.05$ when intact erythrocytes and empty erythrocyte ghosts were compared and when empty erythrocyte ghosts and SD loaded erythrocyte ghosts were compared).

part of encapsulation process, this effect became more pronounced and SD loaded porcine erythrocyte ghosts were losing hemoglobin slower than the corresponding empty ghosts. The difference in the hemoglobin content between SD loaded and empty erythrocyte ghosts became statistically significant after the fourth washing out step and amounted about $20 \%$ on average (Fig. 1C).

It is known that SD exerts biphasic effect on hemolysis: it stabilizes membrane at lower concentrations while causing hemolysis at higher concentrations (Orhan and Sahin, 2001). In this study SD was used in steady state therapeutic concentration (Orhan and Sahin, 2001) which had very mild and delayed antihemolytic effect for porcine erythrocytes. Antihemolytic effect of amphyphiles is well known and extensively described in literature (Sato et al., 1993; Seeman, 1972; Helenius and Simons, 1975; Sanchez et al., 2007). Currently, there are several hypothesis explaining this phenomenon but it requires further detailed studies (Sato et al., 1993; Seeman, 1972; Helenius and Simons, 1975; Sanchez et al., 2007). Regardless the mechanism involved, observed mild and delayed antihemolytic effect of SD did not affect encapsulation process adversely.

It is interesting to note that in the present study SD didn't show antihemolytic effect on the outdated human erythrocytes. These erythrocytes are known to have impaired signalling pathways (Bosman et al., 2008) which together with our finding supports the assumptions that some active mechanism is involved in antihemolytic effect of amphiphiles.

Furthermore, the potassium concentration during the gradual hypotonic hemolysis of porcine erythrocytes was measured with and without presence of SD. As it may be observed from Fig. 1B slight increase in potassium efflux was detected in the presence of SD between 11th and 17th minute. However, the difference between the samples was not statistically significant $(p>0.05)$.
Increased potassium efflux is the common effect shared by the most amphiphiles (Isomaa et al., 1986). According to Isomaa et al. (Isomaa et al., 1986) amphiphiles increase the permeability of the membrane lipid bilayer to the small ions, such as potassium, by decreasing order of packing of the hydrocarbon chains of the lipids in the bilayer.

Number-based particle hydrodynamic diameter distribution curves and statistical measures of the porcine and outdated human erythrocytes, erythrocyte ghosts and erythrocyte ghosts loaded with SD are shown in Fig. 2. The results confirmed that porcine erythrocytes were smaller than human erythrocytes. Porcine ghosts with and without SD were also smaller than the corresponding outdated human samples. It is obvious from the statistical data that the empty ghosts and ghosts loaded with SD were unidispersed introducing important property for their potential application as drug carriers. After the gradual hypotonic hemolysis process and washing out procedure, porcine empty erythrocyte ghosts were on average about $13 \%$ smaller that the intact erythrocytes. On the contrary, outdated human empty erythrocyte ghosts showed an increase in size for about $10 \%$ in comparison with erythrocytes. Subsequently applied encapsulation process caused an increase in ghosts' size of both examined species. Porcine erythrocyte ghosts loaded with SD were about 9\% larger than the empty ghosts but still smaller than starting erythrocytes. Outdated human erythrocyte ghosts loaded with SD were approximately $14 \%$ larger than empty ghosts and larger than starting erythrocytes.

The decrease in erythrocytes' size in gradual hypotonic hemolysis process has been previously reported for porcine erythrocytes (Kostić et al., 2014). Montes et al. (Montes et al., 2008) showed threefold smaller human erythrocyte ghosts than the original cells using isolation by classical hemolysis, i.e. osmotic shock. In the present study where an increase in the size of the ghosts was 


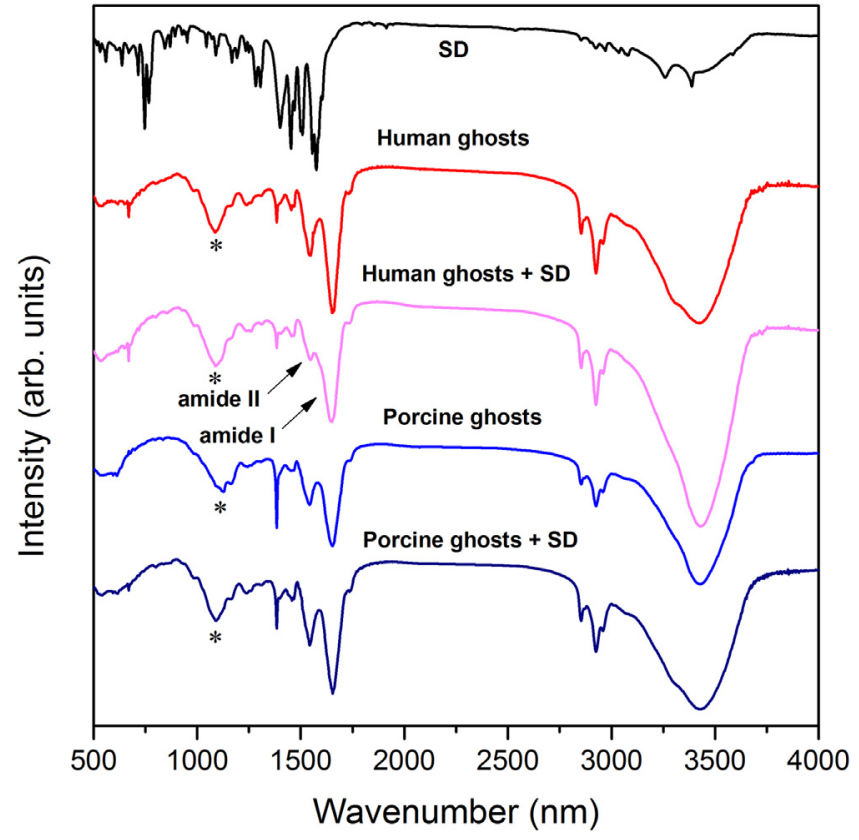

Fig. 3. FTIR spectra of sodium diclofenac (SD), human erythrocyte ghosts, human erythrocyte ghosts loaded with SD, porcine erythrocyte ghosts, porcine erythrocyte ghosts loaded with SD. (Asterisk-a band corresponding to the symmetric $\mathrm{PO}_{2}$ stretching mode).

detected, outdated human erythrocytes obviously lost ability to shrink. On the other hand, porcine erythrocyte ghosts retained size smaller than the original cells. Surface active drugs as well as non-steroidal anti-inflammatory compounds are reported to self-associate and bind to membranes causing the disruption and solubilisation as in detergent-like way (Lopes et al., 2004). Lopes and co-workers showed that SD associates with the lipid bilayer of liposome causing its destruction and the decrease in size (Lopes et al., 2004). This phenomenon was not observed in our study. Erythrocyte ghosts which contain spectrin-actin network (membrane skeleton) in addition to the lipid bilayer (Bennett and Stenbuck, 1979), seem to be resistant to SD's surface active property offering the advantage in comparison with liposomes.

Possible interactions between SD and erythrocyte ghosts as well as changes induced by the applied encapsulation process were investigated by FT-IR (Fig. 3).

The spectra of SD had been described by Bouchal et al. (Bouchal et al., 2016). In the high frequencies range signals due to $\mathrm{N}-\mathrm{H}$ stretching were found at 3382 and $3258 \mathrm{~cm}^{-1}$ (Fig. 3). Signals due to $\mathrm{C}-\mathrm{H}$ stretching in the aliphatic and aromatic part of molecule were found in $3100-2850 \mathrm{~cm}^{-1}$ range (Bouchal et al., 2016). Peaks at 1572 and $1401 \mathrm{~cm}^{-1}$ belong to asymmetric and symmetric stretching modes of carboxylate group, respectively (Bouchal et al., 2016). Signals in $1603-1586 \mathrm{~cm}^{-1}$ range are the consequence of $\mathrm{NH}$ deformation modes and ring stretching modes (Bouchal et al., 2016). Lower frequency range consists of $\mathrm{C}-\mathrm{N}$ stretching modes $\left(1300-1200 \mathrm{~cm}^{-1}\right)$, in plane $\left(1200-1000 \mathrm{~cm}^{-1}\right)$ and out of plane (900-690 $\left.\mathrm{cm}^{-1}\right)$ CH deformations (Bouchal et al., 2016).

The analysis of IR spectra of porcine and human erythrocyte ghosts not loaded with SD (Fig. 3 ) revealed the presence of amide I region predominantly containing $\alpha$-helix structure at $1656 \mathrm{~cm}^{-1}$ and amide II region at $1543 \mathrm{~cm}^{-1}$ (Dong et al., 1990). The relative intensity decrease of amide II band compared to amide I in the case of outdated human erythrocyte ghosts loaded with SD indicates change in the tertiary structure evoked by the presence of SD.

According to Casal and Mantsch phospholipid infrared bands in the spectrum can be divided into three different regions corre-
Table 1

Values of zeta $(\zeta)$-potential and conductivity $(\sigma)$ for porcine and human erythrocyte samples.

\begin{tabular}{llc}
\hline Sample & $\zeta$-potential $(\mathrm{mV})$ & $\sigma(\mathrm{mS} / \mathrm{cm})$ \\
\hline $\begin{array}{l}\text { Porcine erythrocytes } \\
\begin{array}{l}\text { Porcine erythrocyte } \\
\text { ghosts }\end{array}\end{array}$ & $-9.4 \pm 0.61$ & $15.9 \pm 0.66$ \\
$\begin{array}{l}\text { Porcine erythrocyte } \\
\text { ghosts loaded with SD }\end{array}$ & $-10.1 \pm 0.12$ & $15.6 \pm 0.66$ \\
$\begin{array}{l}\text { Human erythrocytes } \\
\text { Human erythrocyte } \\
\text { ghosts }\end{array}$ & $-12.5 \pm 0.89$ & $15.7 \pm 0.50$ \\
$\begin{array}{l}\text { Human erythrocyte } \\
\text { ghosts loaded with SD }\end{array}$ & $-11.7 \pm 0.59^{\mathrm{a}}$ & $15.7 \pm 0.58$ \\
\hline a Significant difference in $\zeta$-potential value of the human erythrocytes.
\end{tabular}

sponding to the hydrophobic, interfacial and polar moieties of the lipid (Casal and Mantsch, 1984). C-H stretching vibrations give rise to bands in the hydrophobic $3100-2800 \mathrm{~cm}^{-1}$ region (Mantsch and McElhaney, 1991). As it may be observed from Fig. 3, asymmetric and symmetric $\mathrm{CH}_{2}$ bands at 2926 and $2852 \mathrm{~cm}^{-1}$, respectively, which are the strongest ones in a phospholipid spectrum (Mantsch and McElhaney, 1991) are clearly observable in porcine and human erythrocyte ghosts, as well as in their SD loaded forms. Shifting of the symmetric $\mathrm{CH}_{2}$ band toward higher frequencies that is according to Szalontai et al. (Szalontai et al., 2000) attributed to the 'structural' disorder imposed on the lipids was not detected after the applied encapsulation process. The most prominent band arising from the interfacial region is due to stretching vibrations of the carbonyl group involved in ester bonds and it is found at $1730 \mathrm{~cm}^{-1}$ and $1737 \mathrm{~cm}^{-1}$ for porcine and human ghosts, respectively (Mantsch and McElhaney, 1991). This band that is very responsive to changes in its environment (Mantsch and McElhaney, 1991), is found at the same positions in the corresponding SD loaded porcine and human ghosts.

The antisymmetric $\mathrm{PO}_{2}{ }^{-}$stretching mode (Mantsch and McElhaney, 1991) appeared at $1245 \mathrm{~cm}^{-1}$ in both porcine and human empty and SD loaded ghosts. The symmetric $\mathrm{PO}_{2}{ }^{-}$stretching mode (Mantsch and McElhaney, 1991) appeared at $1085 \mathrm{~cm}^{-1}$ in human ghosts and was slightly shifted to $1093 \mathrm{~cm}^{-1}$ after the applied encapsulation process (signed with asterisk). Also, this mode was observed at $1121 \mathrm{~cm}^{-1}$ in porcine ghosts but was shifted to $1092 \mathrm{~cm}^{-1}$ in SD loaded ghosts (signed with asterisk). The band shift observed for the symmetric $\mathrm{PO}_{2}{ }^{-}$stretching bands suggest that the SD might be located close to the phosphate moiety of lipid molecules. This finding is in agreement with the study which has previously shown that SD has a potential to interact with phosphate of phosphatidylcholine head groups in liposomes (Lopes et al., 2004). Comparing the spectra of porcine and human empty erythrocyte ghosts with ghosts loaded with SD, no new absorption peaks appeared.

Table 1 shows the values of zeta $(\zeta)$-potential and conductivity $(\sigma)$ of the examined porcine and human erythrocyte samples. As it may be observed, gradual hypotonic hemolysis and encapsulation process didn't affect significantly surface charge and conductivity of the starting porcine erythrocyte suspension. The maintenance of this feature is important for two reasons: (1) when the expression of sialic acid in the erythrocyte surface is reduced, the electrostatic repulsive force decreases and erythrocyte aggregation is accelerated (Shiga et al., 1990), (2) negatively charged cells represent a good template core for layer by layer coating (Shaillender et al., 2011). Statistically significant difference in $\zeta$-potential between human erythrocytes and erythrocyte ghosts $(p<0.05)$ indicates increased loss of $\mathrm{N}$-acetylneuraminic acid probably caused with an aging process potentiated in the seven week storage conditions (Huang et al., 2011). A slight decrease in the surface charge of both porcine and outdated human SD loaded erythrocyte ghosts 

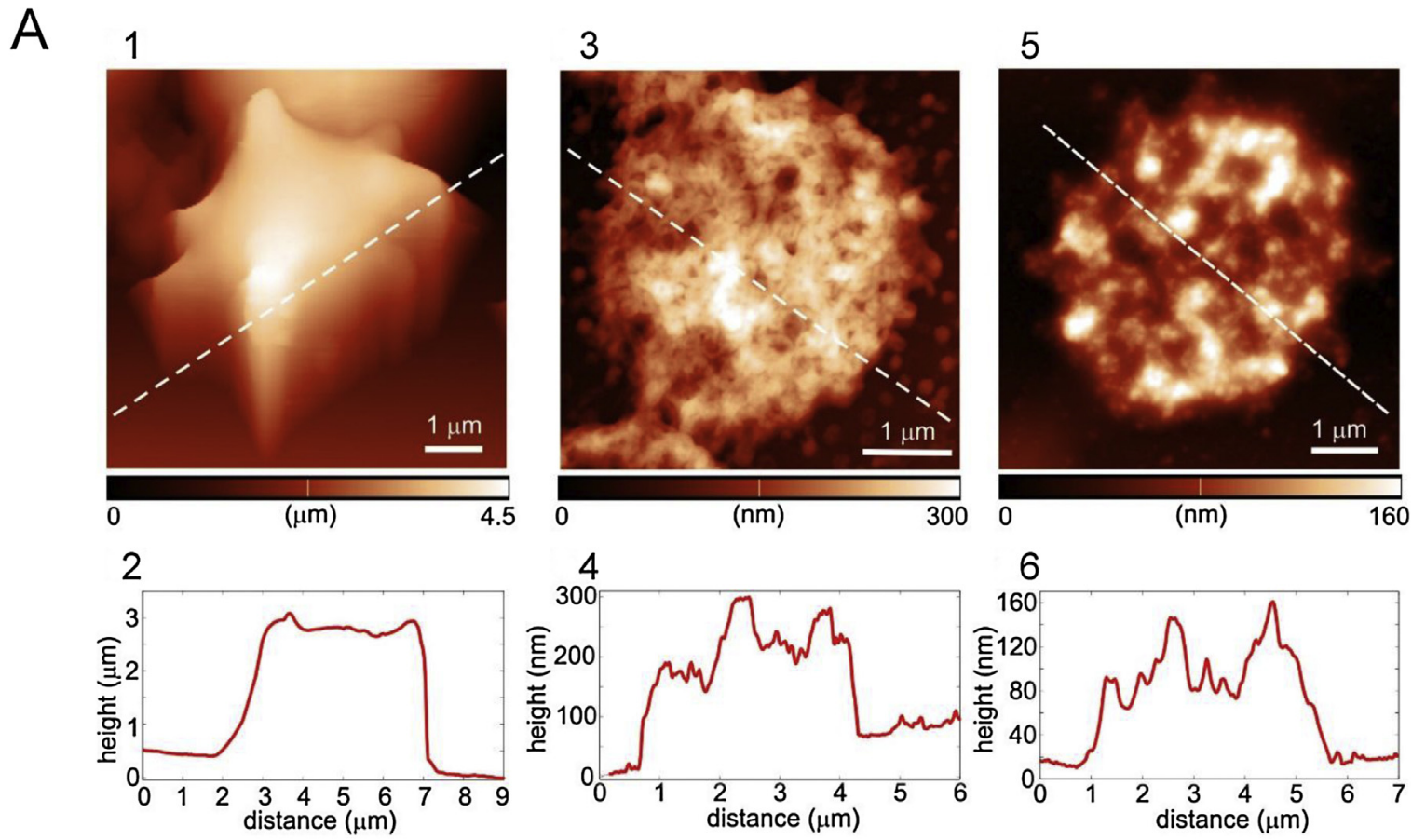

B

1
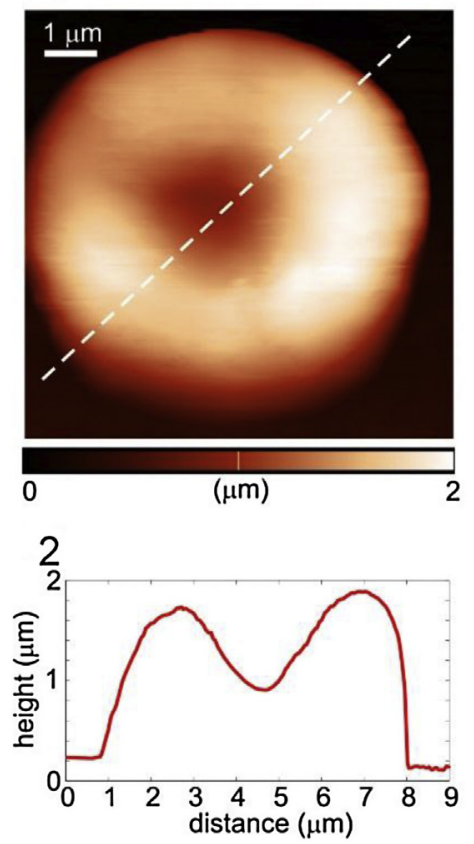

3
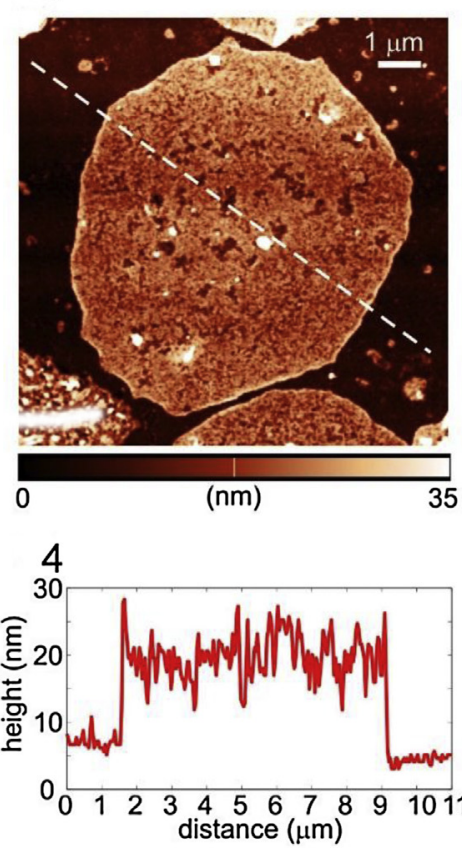

5

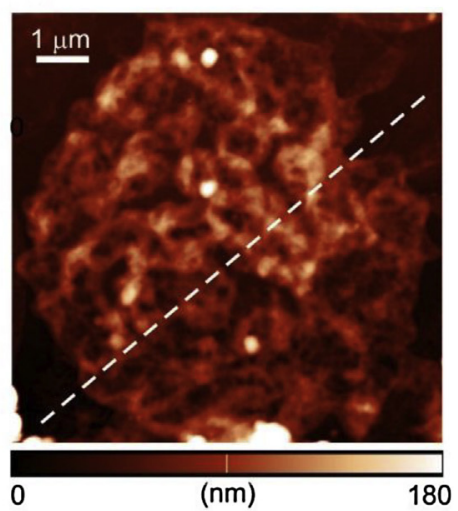

6

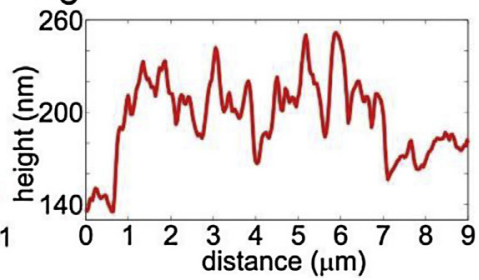

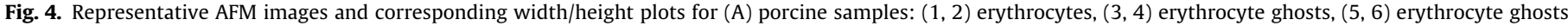
loaded with SD and (B) human samples: $(1,2)$ erythrocytes, $(3,4)$ erythrocyte ghosts, $(5,6)$ erythrocyte ghosts loaded with SD.

in comparison with the corresponding empty ghosts and intact erythrocytes might be a contribution of the negatively charged SD attached to the membrane surface.

Zhbanov et al. (Zhbanov and Yang, 2015) showed the increase of blood conductivity due to aggregation of erythrocytes both theoretically and experimentally. Since changes regarding conductivity were not detected, this result might be a confirmation for the low aggregation ability of the SD loaded ghosts in the final formulation.

Atomic force microscopy revealed significant difference between isolated porcine and outdated human erythrocytes' morphology (Fig. 4A1, 4B1). As it may be observed, starting porcine erythrocytes had echynocytic morphology (Fig. 4A1), contrary to the biconcave morphology of outdated human erythrocytes (Fig. 4B1). Gradual hypotonic hemolysis induced significant distortion in erythrocyte shape and altered surface texture in both species. Additionally, nanoscale alterations and damages were noted in empty erythrocyte ghosts originating from outdated human erythrocytes. Surface roughness values for empty porcine and outdated human erythrocyte ghosts amounted $37 \pm 3 \mathrm{~nm}$ and $7 \pm 2 \mathrm{~nm}$, respectively. After the applied encapsulation process $R_{\mathrm{rms}}$ 


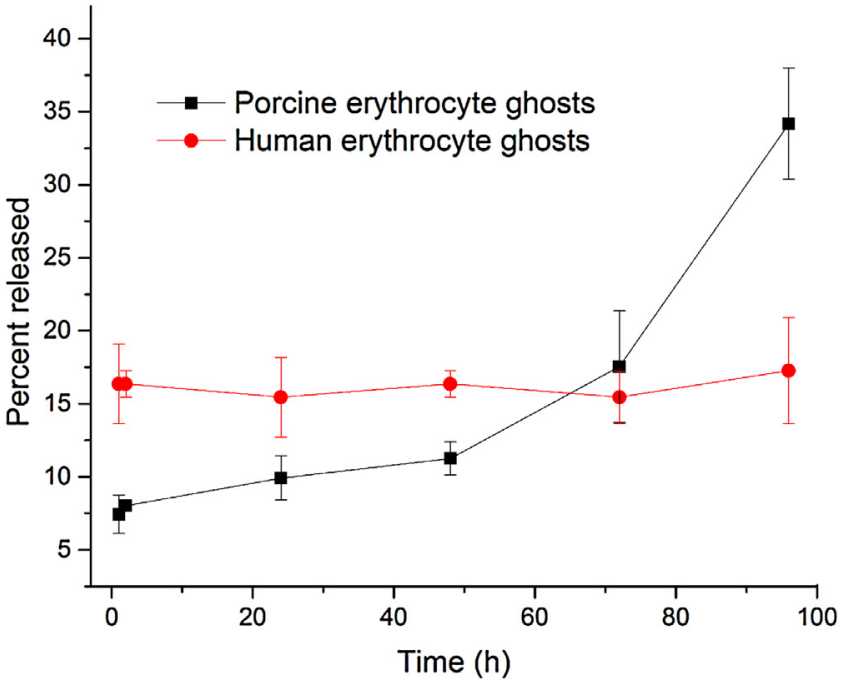

Fig. 5. Release profile of SD from porcine and outdated human erythrocyte ghosts as a function of time.

surface roughness value did not change significantly for porcine sample and it amounted $33 \pm 9 \mathrm{~nm}$. On the other hand, $R_{\mathrm{rms}}$ surface roughness value of SD loaded outdated human erythrocyte ghosts increased to $23 \pm 4 \mathrm{~nm}$. Since erythrocytes are not flat surfaces and curvature effect might affect surface roughness values, they were not compared with empty and SD loaded ghosts.

The echynocyte morphology of porcine erythrocytes represents a common artefact in handling of porcine blood (Barger, 2010). Even though it was expected that outdated human erythrocytes would also have echinocyte morphology due to in vitro aging during the storage (Girasole et al., 2010), they had "normal" shape of flattened biconcave disc. The observed nanoscale damages on the outdated human erythrocyte ghosts indicated their impaired membraneskeleton integrity and higher sensitivity in comparison with the fresh porcine erythrocytes. The damages of the outdated human erythrocytes might be one of the reasons for relatively high level of SD leakage during the washing out procedure and low level of SD encapsulation. Erythrocyte membrane roughness is a morphological parameter that links together the development of nanoscale alterations on the cell membrane with an occurrence of membraneskeleton defects (Girasole et al., 2010). In the case of porcine erythrocyte ghosts, crosslinking of spectrin with glutaraldehyde during the encapsulation process (Shiga et al., 1990) seems to 'improve' surface properties making the membrane smoother. On the other hand SD loaded outdated human erythrocyte ghosts had higher $\mathrm{R}_{\mathrm{rms}}$ values in comparison with the corresponding empty membranes which indicates further rearrangement and perturbations of the surface induced by the encapsulation process. During storage the most central biochemical change is a decrease in ATP which is important in the maintenance of membrane structure and integrity (Bosman et al., 2008). Due to its loss, erythrocytes become effete (Bosman et al., 2008), so higher susceptibility of the outdated human samples to the surface roughness changes was not surprising.

Data given in Fig. 5 show that SD loaded porcine and outdated human erythrocyte ghosts had different drug releasing profiles. Porcine erythrocyte ghosts showed very slow initial release with only $8 \%$ of the drug released within the $2 \mathrm{~h}$, followed by $35 \%$ of the encapsulated SD released after $96 \mathrm{~h}$. On the other hand, outdated human erythrocyte ghosts initially released about two fold higher amount $(\sim 16 \%)$ of the encapsulated SD within $2 \mathrm{~h}$ in comparison with porcine ghosts. The established steady-state of SD between human erythrocyte ghosts and the buffer solution used for the release test within $1 \mathrm{~h}$, has not changed significantly during the next $96 \mathrm{~h}$.

Porcine erythrocyte ghosts didn't show undesirably high burst release which may lead to toxicity and wastage of an encapsulated agent (Shaillender et al., 2011). The amount of released drug within $2 \mathrm{~h}$ from porcine ghosts was much lower in comparison with the other reported SD controlled drug delivery systems such as solid lipid nanoparticles (Liu et al., 2014), chitosan spheres (Kozakevych et al., 2013), PLGA-nanoparticles (Kozakevych et al., 2013) and rat erythrocyte ghosts (Jain and Vyas, 1994). In addition to the lower burst release, porcine ghosts had slower release rate in comparison with the previously mentioned systems. These findings indicate that the porcine erythrocyte membranes derived from the slaughterhouse blood are minimally porous and responded very well on the exposure to the resealing hypertonic solution which is intended to cause shrinkage during the encapsulation process. This is in accordance with the retained ability of porcine ghosts to shrink reflected through the smaller size of SD loaded ghosts in comparison with the starting erythrocytes. Membrane holes successfully closed along with cross-linked membrane proteins with $0.01 \%$ glutaraldehyde were suitable for achievement of slow and extended (prolonged) SD release profile where release rate was based on equilibrium of released and drug retained within ghosts.

On the other hand, SD loaded human erythrocyte ghosts prepared from the outdated erythrocytes with impaired structure and function due to aging during the storage (Huang et al., 2011) showed entirely different SD release pattern from porcine ghosts. As it was shown in Fig. 5, during the in vitro drug release testing, an increase in SD concentration was not detected with time indicating zero-order drug release. SD which probably interacted with the membrane (but was not entrapped inside the membranes due to proven loss of ability to shrink) was released in the medium to the moment when the concentration equilibrium was established. This was in agreement with assumed mechanism of SD retaining in outdated human ghosts by FTIR analysis. According to Briones et al. who obtained similar drug releasing profile for zidovudine in rat erythrocytes, this type of behaviour allows the system to work as a drug reservoir, providing sustained release into the body (Briones et al., 2010).

After comparing encapsulation efficiency of fresh porcine erythrocytes obtained in this study with data on those of fresh human erythrocytes found in the scientific literature, we assumed that preserved structure and function of the membranes seem to be a prerequisite for their usage in encapsulation process

\section{Conclusions}

Although SD due to its surface active properties may bind to membranes and cause its disruption and solubilisation as in detergent-like way, under the applied process based on gradual hemolysis it was successfully encapsulated in erythrocyte ghosts. Despite its antihemolytic effect at lower concentrations, relatively high encapsulation efficiency was achieved. Comparing the two inexpensive, widely available materials-slaughterhouse porcine and outdated transfusion human blood, we demonstrated more preserved structure and functional properties of porcine erythrocytes related to the superior encapsulation and release of SD, and defined them as more promising candidate for the usage in SD encapsulation process on a large scale. However, obtained results indicate the necessity of further investigation in order to produce universal effective SD carrier based on erythrocyte ghosts. These approaches depending of the administration route will include layering with different polymers (in order to mask the antigen determinants on the surface and to control relase more subtle), as well as examination of their behaviour in vivo. 
The present study offers a potential solution for the problem of slaughterhouse waste disposal and on the other hand it contributes to the defining of the optimal formulation for diclofenac.

\section{Acknowledgements}

This work has been supported by Ministry of Education, Science and Technological Development of the Republic of Serbia (Project No. III 46010 and OI 171005). The authors would like to thank Dr. Smilja Marković of the ITS SASA for performing particle size measurements. The authors also thank to Saša Novaković having kindly provided us blood samples from the slaughterhouse PKB "Imes".

\section{Appendix A. Supplementary data}

Supplementary data associated with this article can be found, in the online version, at http://dx.doi.org/10.1016/j.jbiotec.2016.10. 017.

\section{References}

Barger, M.A., 2010. Schalm's Veterinary Haemathology. In: Weiss, J.D., Wardrop, K.J. (Eds.), 6th ed. Blackwell Publishing Ltd, Ames, pp. 144-151.

Bennett, V., Stenbuck, P.J., 1979. Identification and partial purification of ankyrin, the high affinity membrane attachment site for human-erythrocyte spectrin. J. Biol. Chem. 254, 2533-2541.

Bosman, G.J.C.G.M., Were, J.M., Willekens, F.L.A., Novotny, V.M.J., 2008. Erythrocyte aging in vivo and in vitro: structural aspects and implications for transfusion. Transfus. Med. 18, 335-347.

Bouchal, R., Miletto, I., Thach, U.D., Prelot, B., Berlier, G., Hesemann, P., 2016. Ionosilicas as efficient adsorbens for the separation of diclofenac and sulindac from aqueous media (supplementary material). New J. Chem., http://dx.doi. org/10.1039/C6NJ01473A.

Briones, E., Colino, C.I., Lanao, J.M., 2010. Study of the factors influencing the encapsulation of zidovudine in rat erythrocytes. Int. J. Pharm. 401, 41-46.

Carson, J., Notis, W.M., Orris, E.S., 1990. Colonic ulceration and bleeding during diclofenac therapy. Engl. J. Med. 323, 135

Casal, H.L., Mantsch, H.H., 1984. Polymorphic phase behavior of phospholipid membranes studied by infrared spectroscopy. Biochim. Biophys. Acta 779 $381-401$.

Cooper D, L., Harirforoosh, S., 2014. Design and optimization of PLGA-based diclofenac loaded nanoparticles. PLoS One, 9.

Dong, A., Huang, P., Caughey, W.S., 1990. Protein secondary structures in water from second-derivative amide I infrared spectra. Biochemistry 29, 3303-3308.

Doucet, J., Gao, Z., MacLaren, L.A., McAlister, V.C., 2004. Modification of xenoantigens on porcine erythrocytes for xenotransfusion. Surgery 135 $178-186$.

Fun, W., Yan, W., Hu, Z., Ni, H., 2012. Erythrocytes load of low molecular weight chitosan nanoparticles as a potential vascular drug delivery system. Colloids Surf. B 95, 258-265.

Girasole, M., Pompeo, G., Cricenti, A., Longo, G., Boumis, G., Ballelli, A., Amiconi, S., 2010. Nanomed. The how, when, and why of the aging signals appearing on the human erythrocyte membrane: an atomic force microscopy study of surface roughness. Nanotechnol. Biol. Med. 6, 760-768.

Hamidi, M., Tajerzadeh, H., 2001. In vitro characterization of human intact erythrocytes loaded by enalaprilat. Drug Deliv. 8, 223-230.

Hamidi, M., Azimi, K., Mohammadi-Samani, S., 2011. Co-encapsulation of a drug with a protein in erythrocytes for improved drug loading and release: phenytoin and bovine serum album. J. Pharm. Pharm. 14, 46-59.

Helenius, A., Simons, K., 1975. Solubilization of membranes by detergens. Biochim. Biophys. Acta 415, 29-79.

Huang, Y.H., Wu, Z.J., Mehrishi, J., Huang, B.T., Chen, H.J., Zheng, H.J., Liu, W.J., Luo, M., 2011. Human red blood cell aging: correlative changes in surface charge and cell properties. J. Cell. Mol. Med. 15, 2634-2642.

Isomaa, B., Hagerstrand, H., Paatero, G., Engblom, A.C., 1986. Permeability alterations and antihaemolysis induced by amphiphiles in human erythrocytes. Biochim. Biophys. Acta 860, 510-524.

Jain, S.K., Vyas, S.P., 1994. Magnetically responsive diclofenac sodium-loaded erythrocytes: preparation and in vitro characterization. J. Microencapsulation $11,141-151$.
Kostić, I., Ilić, V., Đorđević, V., Bukara, K., Mojsilović, S., Nedović, V., Bugarski, D., Veljović, Đ., Mišić, D., Bugarski, B., 2014. Erythrocyte membranes from slaughterhouse blood as potential drug vehicles: isolation by gradual hypotonic hemolysis and biochemical and morphological characterization. Colloids Surf. B 122, 250-259.

Kozakevych, R.Z., Bolbykh, Y.M., Tertykh, V.A., 2013. Controlled release of diclofenac sodium from silica-chitosan composites. World J. Nanosci. Eng. 3, 69-78.

Krajišnik, D., Daković, A., Malenović, A., Rakić, M.M., Dondur, V., Radulović, Ž., Milić, J., 2013. Investigation of adsorption and release of diclofenac sodium by modified zeolite composites. Appl. Clay Sci. 83, 322-326.

Kulkarni, A.R., Kumaresh, S., Soppimath, Soppimath Aminabhavi, T.M., 1999. Controlled release of diclofenac sodium from sodium diclofenac beads crosslinked with glutaraldehyde. Pharm. Acta Helv. 74, 29-36.

Liu, D., Chen, L., Jiang, S., Zhu, S., Quian, Y., Wang, F., Li, R., Xu, Q., 2014. Formulation and characterization of hydrophilic drug diclofenac sodium-loaded solid lipid nanoparticles based on phospholipid complexes technology. J. Liposome Res. 24, 17-26.

Lopes, L.B., Scarpa, M.V., Silva, G.V.J., Rodrigues, D.C., Santilli, C.V., Oliviera, A.G., 2004. Studies on the encapsulation of diclofenac in small unilamellar liposomes of soya phosphatidylcholine. Colloids Surf. B 39, 151-158.

Mansouri, S., Merhi, Y., Winnik, F.M., Tabrizian, M., 2011. Investigation of layer-by-layer assembly of polyelectrolytes on fully functional human red blood cells in suspension for attenuated immune response. Biomacromolecules 12, 585-592.

Mantsch, H.H., McElhaney, 1991. Phospholipid phase transitions in model and biological membranes as studied by infrared spectroscopy. Chem. Phys. Lipids 57, 213-226.

Mao, Z., Cartier, R., Hohl, A., Farinacci, M., Dorhoi, A., Nguyen, T.L., Mulvaney, P., Ralston, J., Kaufmann, S.H.E., Möhvald, H., Wang, D., 2011. Cells as factories for humanized encapsulation. Nano Lett. 11, 2152-2156.

Millan, C.G., Marinero, M.L.S., Castaneda, A.Z., Lanao, J.M., 2004. Drug, enzyme and peptide delivery using erythrocytes as carriers. J. Controlled Release 95, 27-49.

Montes, L.R., Lopes, D.J., Sot, J., Bagatolli, L.A., Stonehouse, M.J., Vasil, M.L., Wu, B.X. Hannun, Y.A., Goni, F.M., Alonso, A., 2008. Ceramide-enriched membrane domains in red blood cells and the mechanism of sphingomyelinase-Induced hot-cold hemolysis. Biochemistry 47, 11222-11230.

Oddsson, E., Gudjonsson, H., Thjodleifsson, B., 1990. Endoscopic findings in the stomach and duodenum after treatment with enteric-coated and plain naproxen tablets in healthy subjects. Scand. J. Gastroenterol. 25, 231-234.

Orhan, H., Sahin, G., 2001. In vitro effects of NSAIDS and paracetamol on oxidative stress-related parameters of human erythrocytes. Exp. Toxic. Pathol. 53, 133-140.

Sanchez, L., Martinez, V., Infante, M.R., Mitjans, M., Vinardell, M.P., 2007. Hemolysis and antihemolysis induced by amino acid-based surfactants. Toxicol. Lett. 169 $177-184$.

Sato, Y., Yamakose, H., Suzuki, Y., 1993. Participation of band 3 protein in hypotonic hemolysis of human erythrocytes. Biol. Pharm. Bull. 16, 188-194.

Seeman, P., 1972. The membrane action of anesthetics and tranquilizers. Pharmacol. Rev. 24, 583-655.

Shaillender, M., Luo, R., Venkatraman, S.S., Neu, B., 2011. Layer-by-layer microcapsules template on erythrocyte ghosts carriers. Int. J. Pharm. 415, 211-217.

Shiga, T., Maeda, N., Kon, K., 1990. Erythrocyte rheology. Crit. Rev. Oncol. Hematol. $10,9-48$.

Stojanović, R., Ilić, V., Manojlović, V., Bugarski, D., Dević, M., Bugarski, B., 2012. Isolation of hemoglobin from bovine erythrocytes by controlled hemolysis in the membrane bioreactor. Appl. Biochem. Biotechnol. 166, 1491-1506.

Suwalsky, M., Manrique, M., Villena, F., Sotomayor, C.P., 2009. Structural effects in vitro of the antiinflamatory drug diclofenac on human erythrocytes and molecular models on cell membranes. Biophys. Chem. 141, 34-40.

Szalontai, B., Nishiyama, Y., Gombos, Z., Murata, N., 2000. Membrane dynamics as seen by Fourier transform infrared spectroscopy in a cyanobacterium, Synechocystis PCC 6803. The effect of lipid unsaturation and the protein-to-lipid ratio. Biochim. Biophys. Acta 1509, 409-419.

Todd, P.A., Sorkin, E.M., 1988. Diclofenac sodium: a reappraisal of its pharmacodynamics and pharmacokinetic properties and therapeutic efficiency. Drugs 35, 244-285.

Van Assendelft, O.W., Holtz, A.H., Lewis, S.M., 1984. Recommended Method for the Determination of the Haemoglobin Content of Blood. I.C. S. H. Publications, World Health Organization, 1. 4. 1.

Witham, R., 1991. Voltaren (diclofenac-sodium)-induced ileocholitis. J Gastroenterol. 86, 246-247.

Zhbanov, A., Yang, S., 2015. Effects of aggregation on blood sedimentation and conductivity. PLoS One, 1-25. 